\title{
Modeling and Simulation Capability Development Plan
}

The INL is a

U.S. Department of Energy

National Laboratory

operated by

Battelle Energy Alliance

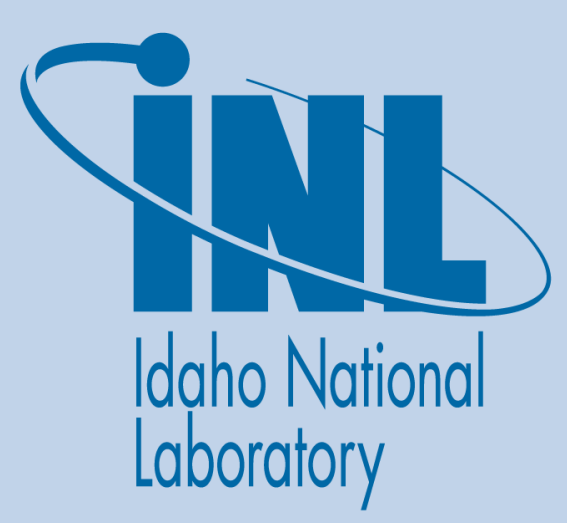

Cristian Rabiti (INL) Aaron Epiney (INL)

Paul Talbot (INL) Jong Suk Kim (INL)

December 2017

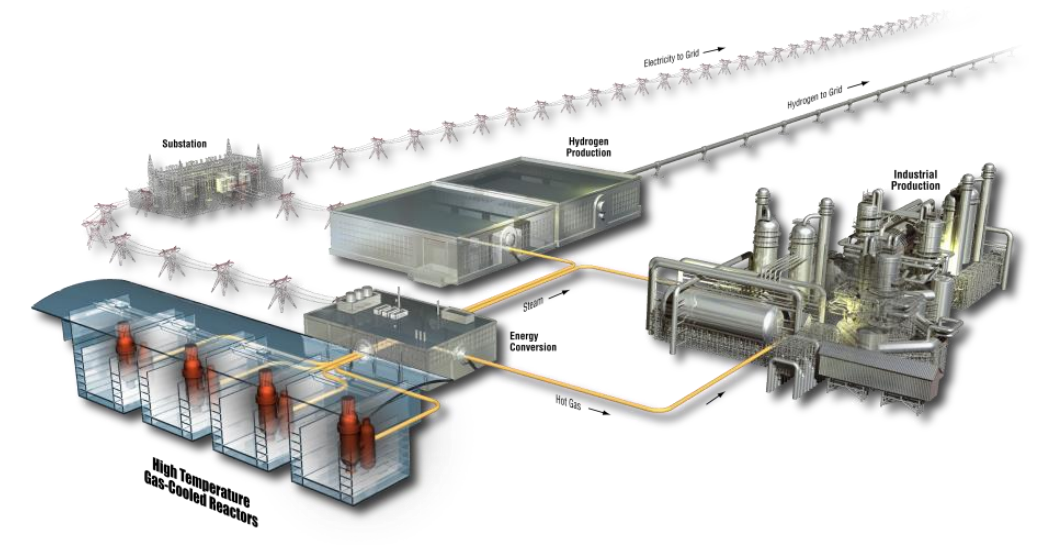




\section{DISCLAIMER}

This information was prepared as an account of work sponsored by an agency of the U.S. Government. Neither the U.S. Government nor any agency thereof, nor any of their employees, makes any warranty, expressed or implied, or assumes any legal liability or responsibility for the accuracy, completeness, or usefulness, of any information, apparatus, product, or process disclosed, or represents that its use would not infringe privately owned rights. References herein to any specific commercial product, process, or service by trade name, trade mark, manufacturer, or otherwise, does not necessarily constitute or imply its endorsement, recommendation, or favoring by the U.S. Government or any agency thereof. The views and opinions of authors expressed herein do not necessarily state or reflect those of the U.S. Government or any agency thereof. 


\title{
Modeling and Simulation Capability Development Plan
}

\author{
Cristian Rabiti (INL) \\ Aaron Epiney (INL) \\ Paul Talbot (INL) \\ Jong Suk Kim (INL)
}

December 2017

Idaho National Laboratory

Idaho Falls, Idaho 83415

http://www.inl.gov

Prepared for the

U.S. Department of Energy

Office of Nuclear Energy

Under DOE Idaho Operations Office

Contract DE-AC07-05ID14517 



\section{CONTENTS}

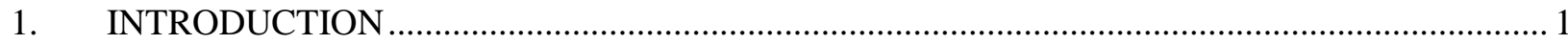

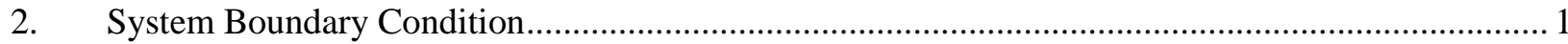

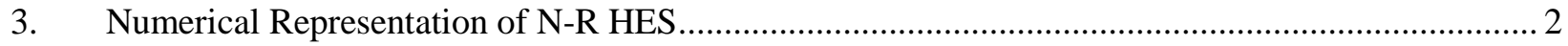

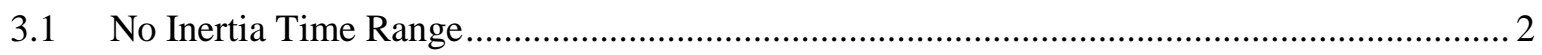

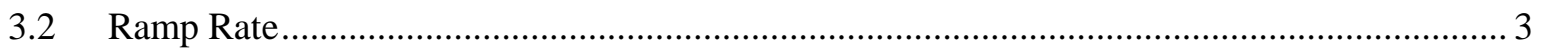

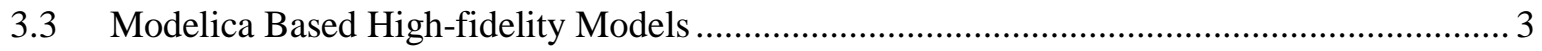

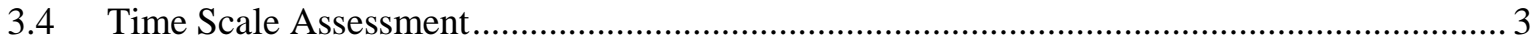

3.5 Improvement of the Cash Flow Module ........................................................................ 4

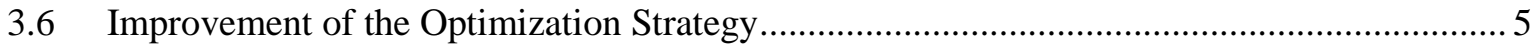

3.6.1 Degradation of the performance for large parallel simulations................................... 6

3.6.2 Long computational time for high fidelity based optimization................................ 6

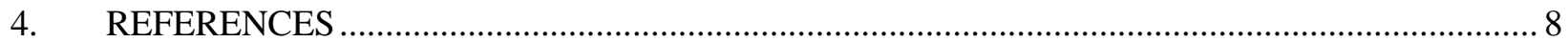




\section{FIGURES}

Figure 1: Simulation workflow for the optimization of N-R HES .................................................... 1

Figure 2: Qualitative expected behavior of the simulation error at different time scales for

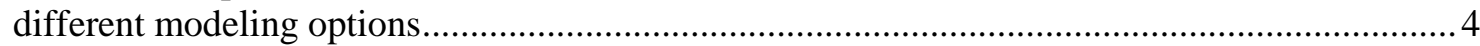

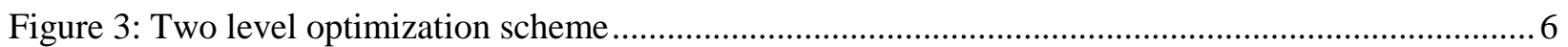




\section{Modeling and Simulation Capability Development Plan}

\section{INTRODUCTION}

This report provides an assessment of the current status of the modelling and simulation capability for the Nuclear-Renewable Hybrid Energy System (N-R HES).

The current capabilities are separated in:

- Creation of the system boundary condition. More in detail this refer to the capability to create either demand, renewable supply, price profiles etc..

- Creation of a numerical representation of the physical model of a N-R HES system

- Derivation, from the actual performance of the physical system, the cash flow to perform the economical assessment

- Optimization of N-R HES configuration and dispatch

Those capabilities are the ones which enable the workflow summarize in Figure 1below.

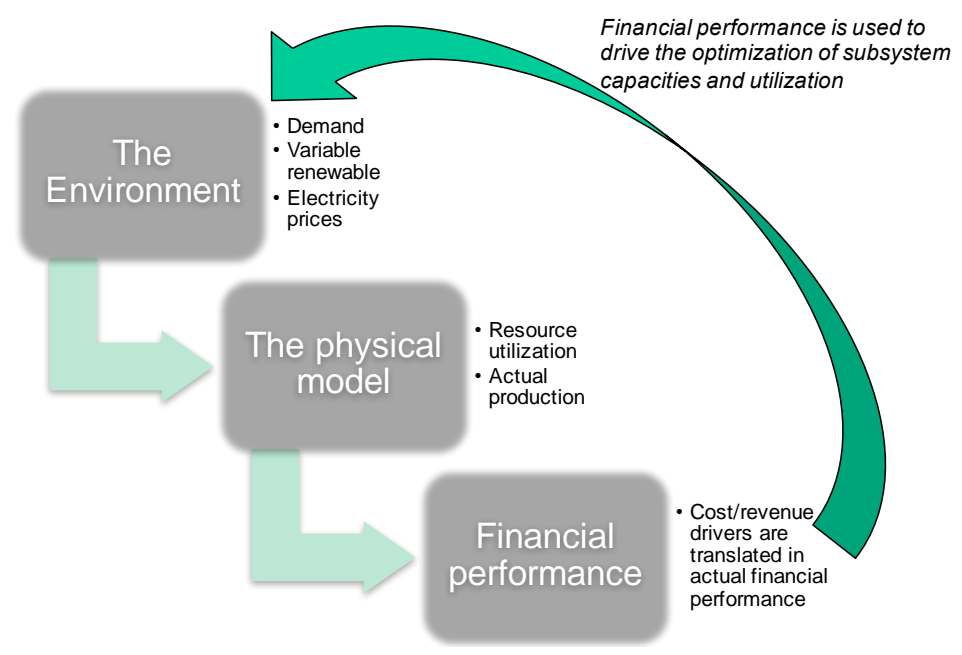

Figure 1: Simulation workflow for the optimization of N-R HES

In the following we will highlight the current status and gap to proceed further

\section{System Boundary Condition}

The creation of system boundary condition refers to the capability to create synthetic data (time histories) representing an unbounded number of realizations similar to the data training set which has a very low cardinality. An example would be the generations of thousands of yearly electricity demand (realizations) with an hourly resolution statistical equivalent to a training set of few years (low cardinality).

Currently the approach used is the one named FARMA (Fourier plus Auto Regressive Moving Average). In this approach, a de-trending of the time series is performed using Fourier while the stochastic auto-correlated component is represented by the ARMA. 
The description of this methodology and its validation (for electricity demand and wind supply) for reconstructing a year of hourly data has been presented in [Jun paper].

The extension of the application range of this methodology to different time resolution, time length and the introduction of new type boundary condition (electricity prices, solar availability) requires to improve and revalidate more extensively the methodology. More in the specific:

1. Creation of guidelines for the choice of the Fourier de-trending frequency as a function of time length (year, monthly, week, day and hourly) and time resolution (hourly, 15 minutes, 5 minutes, one minutes etc.). This task is currently assigned to Universities Space Research Association (USRA)

2. The electricity prices have a much less cyclical behavior than electricity demand (correlation with demand is randomly broken), with unexpected spikes, which exceed one order of magnitude in price change. This is due to phenomena as local grid congestion and supplier unexpected down time. The application of the FARMA methodology needs to be better validated and assessed for this signals. This task is currently assigned to Universities Space Research Association (USRA)

3. The expansion of the boundary condition to include solar electricity supply, it requires to account for the correlation of electricity demand/price with solar availability. To deal with this aspect the FARMA will be expanded to add terms in the autocorrelation matrix to account for correlation cross different time series. This will require the extension of the FARMA model in RAVEN. This task is assigned to INL

\section{Numerical Representation of N-R HES}

At this point three level of fidelity have been recognized needed to achieve the best tradeoff between computational cost and accuracy at different time scales. The differentiation happens depending from the fact that the system is showing or not showing a memory effect. To exemplify, if we consider the hourly time scale, a system would not show inertia if, independently of the initial condition, the system is capable to achieve any allowed output level. The assumption so far made is that there are three-time scale at which the system shows different responses.

A side note is the nuclear plant is not currently considered to assess the characteristic behavior of the N-R HES, since it is not supposed to do load following.

Currently also batteries are not considered to assess the characteristic time scale of the system since their inertia is infinite. There is no

Below the three different behaviors are explained and application ranges suggested

- No inertia. At time scales longer than one hour, we are sure that the system has no memory. Initial testing has shown that depending the composition of the system (different type of plants) inertia might be absent until 15 minutes. This

- Ramp rate. At times below 15 minutes, for sure, the system shows inertia. At the same time, at those time scale (longer than a minute), the achievable system output is bounded by a linear ramp rate.

- Below a minute the system behavior is most likely not linear and a high-fidelity representation of the system is needed to properly capture the system response.

\subsection{No Inertia Time Range}

In the "no inertia" region, no modelling of the physical system is needed. Also, it can be proven that the optimal economical dispatch of the resources is the one which minimize the marginal cost of suppling the next unit of electricity. This is valid either in a profit maximization or in a cost of electricity production minimization. Given these considerations, this situation simplifies a lot the simulation 
requirements, no physical model needed, no optimization needed on the dispatch (dispatchement stack is based on the marginal cost), only system sizing needs to be optmized.

\subsection{Ramp Rate}

In the ramp rate situation, the optimal dispatch is not anymore obtainable by the minimization of the marginal cost, consequently the optimization needs to be run both for optimal dispatch and optimal sizing.

The reason for the marginal cost dispatch not being anymore optimal, is that, for example, demand might deep for a short interval and pick up immediately after. This might generate a situation where it is not the most economic rewarding option to try to follow the load for a component with a lot of inertia.

Bounding ramp rates could be obtained by running the high-fidelity models (modelica based) for different level of initial output of the component to find the maximum achievable delta within the considered time scale. This process has been illustrated in the September milestone [xxx].

Another way to obtain limiting ramp rate is to relay on vendor maximum ramp rate specifications. The ramp rate approach has been used but not so extensively as other approaches and might need some refinement which is currently estimated to be minor.

\subsection{Modelica Based High-fidelity Models}

The system representation based on modelica is the high-fidelity reference representation of the physical system. The list of the component already available is reported in [milestone]. The list of the currently undergoing efforts in this area are reported below:

- Upgrade of the High-Temperature Steam Electrolysis (HTSE) to be compatible with the newest modelica library (INL)

- Update the reverse osmosis desalination plant model to be compatible with the rest of the component of the N-R HES modelica models in the hybrid repository (INL)

- Investigation of random failure of the modelica N-R HES model detected during the September milestone [] by ORNL

- Investigation of model speed up and accuracy respect time scale resolution of the modelica N-R HES model by ANL

\subsection{Time Scale Assessment}

As already mentioned the knowledge of the lower time scale at which the different models (no inertia, ramp rate bounded, modelica) are applicable and their error margin is key in choosing properly using the models for go-no-go decisions.

In Figure 2 it is reported a qualitative expected behavior of the error for the different models at different time scales. No unit of measure are reported on the axis since the figure is just representative of the expected behavior. Increasing the time scale all the models should converge to the same low error, decreasing the time scale the separate with the modelica being the most accurate and the no inertia the less accurate. When taking go-no-go decision the error characteristic of the time scale and the model used should be accounted for. No decision could not be taken if the error exceeds the margin from the go-nogo threshold. 


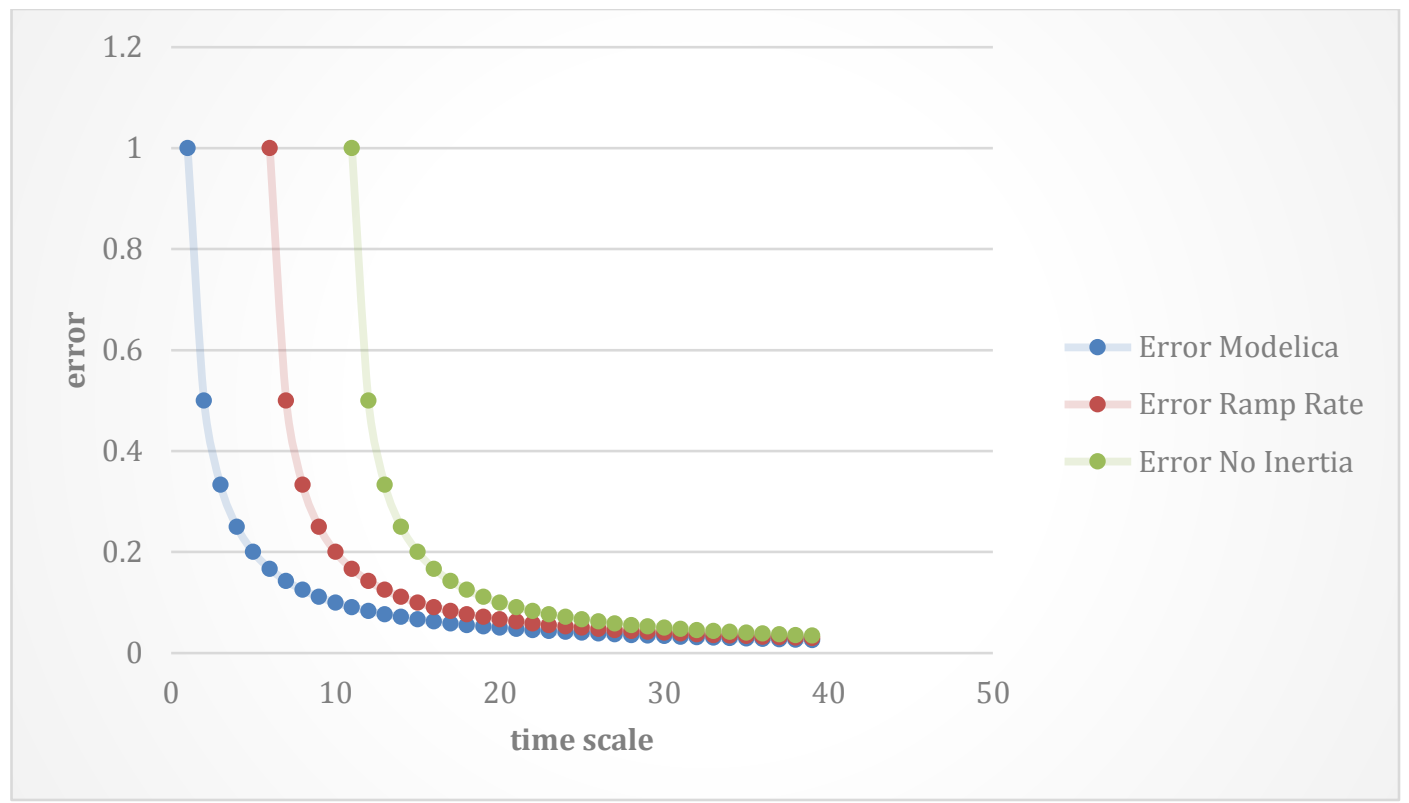

Figure 2: Qualitative expected behavior of the simulation error at different time scales for different modeling options

Replacing the above plot with a quantitative one is one of the task that will be accomplished this year (INL).

\section{Improvement of the Cash Flow Module}

The cash flow module takes as input:

- From its own separate input file:

- fix cost of each subsystem (each single plant) and their scaling factor for changing sizes

- variable cost/revenue and their respective cost/revenue drivers (e.g. gas price associate to gas consumption

- Economic environment definition (e.g. inflation rate, tax rate etc)

- From the system optimization

$\circ$ The size of the subsystems

$\circ$ The actual usage of the resources (cost/revenue) drivers

This module has been just recast as a separate RAVEN plugin, allowing for its independent copyright which will be likely assessed this year.

Minor improvement are expected during this FY with the aim to increase its flexibility. An initial list is reported below:

- Allowing different discount rate for different cash flow to allow to account for different risk profiles and different inflation rates

- Allowing for custom investment sequence to account for already preexisting structure (e.g. already existing nuclear power plant), exceptional CAPEX outside of normal O\&M, and not matching lifetime of investment. 
- More complex scaling function for capital cost to be able to capture phenomenon like transition between economy and diseconomy of scale.

\section{Improvement of the Optimization Strategy}

The optimization strategy is converging towards its final form. Definitively the problem at hand is unique given the very large dimensionality and stochastic behavior.

The final decision made last year was to separate the problem in two components, the optimization of the dispatch and the optimization of the component size. The rationale behind this decision is that the high dimensional component of the problem can now be solved without considering the stochastic component. In fact as it can be seen in Figure 3 the lower level does not contain the environment changes, which means that at the lower level the time histories are not changing anymore at each iteration of the dispatch optimization. The dispatch problem remains a very high dimensional problem (one variable for each time interval and for each component of the N-R HES) and therefore the optimization might be rather slow. To overcome such a challenge a lower order model is used to provide a good guess of the initial guess for the optimal dispatch. Great success has been achieved using the marginal cost based dispatch to accelerate the optimization of the dispatch for a modelica system model. To be notice that the test was performed at a time scale where the marginal cost dispatch was probably already highly accurate. It is possible that in the future for shorter time scale more effective acceleration will be needed. Given the high dimensionality of the problem the optimization of the lower level is driven by a zero order approximation of the gradient, which is very computational cheap per each iteration, being insensitive of the number of dimension, but slower in total number of iteration than a full gradient. Given the number of optimization variables in the thousands the trade-off is in favor of the zero-order approximation

The upper optimization scheme is based on a full gradient (here the number of variable is only the size of the different sub-system) but, given the variation in the environmental variables (electricity demand/price, wind supply etc.) is stochastic. We can describe this algorithm as a variation of the Simultaneous Perturbation Stochastic Approximation which use the full gradient rather than the zeroth order

As already mentioned the overall optimization strategy is fairly mature even if few challenges remain which are being currently addressed and described in the next sections. 


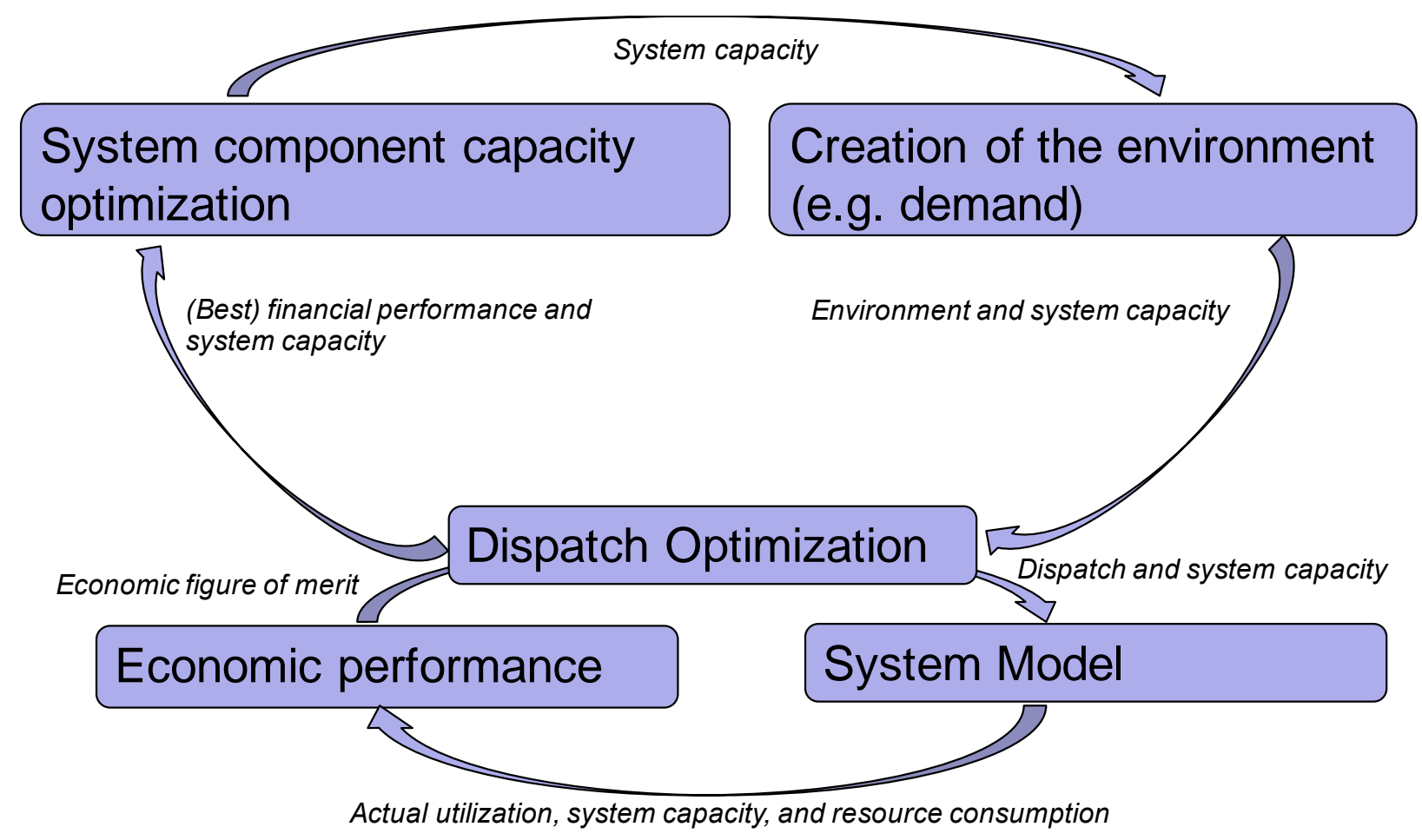

Figure 3: Two level optimization scheme

\subsection{Degradation of the performance for large parallel simulations}

It has been noticed that, while trying to take advantage of a large number of cores (few hundreds) to de-noise the stochastic component of the optimization and to start multiple trajectory to avoid local minimum, the performance of the algorithm was quickly degrading. This has been identified as an issue coming from the data structure used to store the data. This issue has been solved and is currently being tested to verify that the problem has been properly addressed (INL)

\subsection{Long computational time for high fidelity based optimization.}

The modelica based model of the N-R HES takes about two hours to simulate one week of real time. In spite of trying to take advantage of parallel computing (multi trajectory, de-noising) the optimization process degrades fairly quickly in parallel (number of optimization cycle to reach convergence) due to the inherently sequential nature of the process (at each iteration the guess of the next optimal points depends from the previous one). It has been estimated that the optimization process, in spite of how many processor will be used, it will always have a cost equivalent to no less then hundred sequential iterations. This will put the time necessary to perform the optimization for a week of real time, using the high fidelity representation of the system (modelica) at one week of computational time. This is a situation which needs to be address. The following effort are ongoing in this direction:

1. Testing capability of modelica to run in parallel (openMP). This could easily provide a computational saving factor of 1-10 (ORNL)

2. Definition of acceleration schemes based on ramp rate. This would allow to reduce the number of effective equivalent sequential run leading to a saving (when running the highfidelity model) between 1-10 (ANL) 
3. Simplification and increase robustness of modelica model (ANL) which could lead to a saving factor of 2-5

4. Identification of characteristic time intervals. While it is expected a strong reduction of the computational time by the above suggested strategies, extending an high fidelity optimization for an whole year seems to be a stretch. Mainly the reason would be the increase of the dimensionality space so that the gradient of the goal function, with respect for example the dispatch of one of the subsystem at a given hour is practically flat with respect the overall gradient. To avoid this we could identify representative time periods (representative days of the year), perform the optimization only for those days and then combine them back (with the contribution of each day proportional to the number of days it is representative of with respect the full year). There are two difficult steps in this approach:

a. Identification of the representative days (or any other time interval we might choose, e.g. weeks, or hours)

b. Avoid that initial conditions dominate the optimization solution

To avoid the second problem, it will be necessary to take time interval long enough so that the memory of the initial conditions are vanished. This information will be actually available by the analysis performed to determine the memory of each subsystem. As already mentioned, special care will be needed for the battery and the reactor.

Once that the minimum interval is determined, the selection of the representative time intervals will be performed using clustering on the FARMA coefficient which will be used to represent those time intervals. For example, if a week is determined to be the minimum time interval, a number of FARMA equal to the number of week available in the training database will be generated and they will be clustered accordingly the distance in the space of the Fourier moments and covariance matrix entries.

This task could be eventually carried over in FY 19, given that most of the analysis commited this year could be performed with a reasonable degree of accuracy without the full deployment of this methodology. 


\section{REFERENCES}

1. H. Elmqvist, 
